\title{
EVERY NEEDLE POINT SPACE CONTAINS A COMPACT CONVEX AR-SET WITH NO EXTREME POINTS
}

\author{
NGUYEN TO NHU AND LE HOANG TRI
}

(Communicated by James E. West)

\begin{abstract}
Every needle point contains a compact convex AR-set without any extreme points. In particular the following spaces contain such compact convex sets: (i) the spaces $L_{p}, 0 \leq p<1$; (ii) the linear metric space constructed by Roberts (Studia Math. 60 (1977), 255-266).
\end{abstract}

\section{INTRODUCTION}

The following classical results are well known in functional analysis and topology.

1-1. Theorem (Krein-Milman [KM]). Every compact convex set in a locally convex space is the closed convex hull of its extreme points.

1-2. Theorem (Schauder, see [DG]). Every compact convex set in a locally convex space has the fixed point property.

1-3. Theorem (Dugundji, see [BP]). Every convex set in a locally convex linear metric space is an $A R$.

It is natural to ask whether Theorems 1-1-1-3 still hold for nonlocally convex spaces. This question is extremely difficult. In 1976 Roberts [R1] constructed a striking example of a linear metric space which contains a compact convex set without any extreme points. Thus the Krein-Milman theorem does not hold in nonlocally convex spaces.

After Roberts constructed his example the following problem arose.

1-4. Problem. Can Roberts' example be used to provide a counterexample to Schauder's theorem or to Dugundji's theorem for nonlocally convex spaces? (see [G, Problem LS7].)

In this paper we show that some compact convex sets constructed by Roberts are AR, hence are homeomorphic to the Hilbert cube. Our result provides first examples of compact convex sets with no extreme points which are $A R$.

Received by the editors March 28, 1990 and, in revised form, July 20, 1992.

1991 Mathematics Subject Classification. Primary 54C55; Secondary 54D45.

Key words and phrases. Needle point, needle point space, extreme point, convex set, AR, fixed point property, Hilbert cube, admissible convex set. 


\section{THE MAIN RESULT}

Before stating our main result we introduce some notation and conventions which will be used in the sequel.

By a linear metric space we mean a topological linear space $X$ which is metrizable. By Kakutani's theorem (see, for instance, $[R e]$ ) there is an invariant metric $\rho$ on $X$. We denote $\|x-y\|=\rho(x, y)$. Observe that $\|\cdot\|$ is not a norm; in particular $\|\lambda x\|_{i} \neq|\lambda|\|x\|$. However we may assume that $\|\lambda x\| \leq\|x\|$ for every $x \in X$ and $\lambda \in \mathbb{R}$ with $|\lambda| \leq 1$. The zero element of $X$ is denoted by $\theta$.

Let $A$ be a subset of a linear metric space $X$ and $x \in X$. We use the following notation:

$$
\begin{gathered}
A^{+}=\operatorname{conv}(A \cup\{\theta\}) ; \quad \hat{A}=\operatorname{conv}\left(A^{+} \cup\left(-A^{+}\right)\right) ; \\
\|x-A\|=\inf \{\|x-y\|: y \in A\} .
\end{gathered}
$$

Following Roberts [R1, R2] let us say that a nonzero point $a$ of a linear metric space $X$ is a needle point if and only if for every $\varepsilon>0$ there exists a finite set $A=\left\{a_{1}, \ldots, a_{m}\right\}$ satisfying the following conditions:

(1) $\left\|a_{i}\right\|<\varepsilon$ for every $i=1, \ldots, m$;

(2) For every $b \in A^{+}$there is an $\alpha \in[0,1]$ such that $\|b-\alpha a\|<\varepsilon$;

(3) $a=\frac{1}{m}\left(a_{1}+\cdots+a_{m}\right)$.

We say that $X$ is a needle point space if and only if $X$ is a complete linear metric space in which every nonzero point is a needle point. Roberts [R2] has shown that for every $p \in[0,1)$ the space $L_{p}$ is a needle point space. We recall that the spaces $L_{p}, 0 \leq p<1$, are defined by

$$
L_{p}=\left\{f:[0,1] \rightarrow \mathbb{R} ; \int_{0}^{1}|f(t)|^{p} d t<\infty\right\} \quad \text { for } 0<p<1 ;
$$

and

$$
L_{0}=\left\{f:[0,1] \rightarrow \mathbb{R} ; \int_{0}^{1} \frac{|f(t)|}{1+|f(t)|} d t<\infty\right\} .
$$

Our result in this paper is the following:

2-1. Theorem. Every needle point space $X$ contains a compact convex set $C$ without any extreme points such that $C$ is homeomorphic to the Hilbert cube.

Proof. Let $a_{0}$ be a nonzero point of $X$. Using the needle point space property of $X$ we choose by induction a sequence $\left\{A_{n}\right\}$ of finite subsets of $X$ with $A_{0}=\left\{a_{0}\right\}$, a sequence of retractions $r_{n}: X \rightarrow \hat{A}_{n}$ and two sequences of positive numbers $\left\{\varepsilon_{n}\right\}$ and $\left\{\delta_{n}\right\}$ with the following properties:

(4) $\|a\|<\varepsilon_{n}$ for every $a \in A_{n}$;

(5) $\varepsilon_{n}<\frac{1}{8 m(n-1)} \min \left\{\delta_{n-1}, 2^{-n}\right\}$ and $\delta_{n}<\frac{1}{2} \delta_{n-1}$; where $m(n)=\operatorname{Card} A_{n}$;

(6) If $x \in X$ and $\left\|x-\hat{A}_{n}\right\|<\delta_{n}$ then $\left\|x-r_{n}(x)\right\|<\varepsilon_{n}$;

(7) If $A_{n}=\left\{a_{1}^{n}, \ldots, a_{m(n)}^{n}\right\}$ then $A_{n+1}$ can be written in the form

$$
A_{n+1}=\bigcup_{i=1}^{m(n)} A_{n+1}\left(a_{i}^{n}\right)
$$


where $A_{n+1}\left(a_{i}^{n}\right), i=1, \ldots, m(n)$, satisfy the conditions

(7-a) $a_{i}^{n}=t_{i}^{n} \sum_{a \in A_{n+1}\left(a_{i}^{n}\right)} a$, where $t_{i}^{n}=\left(\operatorname{Card} A_{n+1}\left(a_{i}^{n}\right)\right)^{-1}$;

(7-b) for each $a \in A_{n+1}^{+}\left(a_{i}^{n}\right)$ there exists an $\alpha \in[0,1]$ such that $\left\|a-\alpha a_{i}^{n}\right\|<$ $\varepsilon_{n+1}$.

From (7-a) it follows that

(8) $\hat{A}_{n} \subset \hat{A}_{n+1}$ for every $n \in N$.

We show

2.2. Claim. $\left\|x-\hat{A}_{n}\right\|<\frac{1}{4} \delta_{n}$ for every $x \in \hat{A}_{n+1}$.

Proof. For every $x \in \hat{A}_{n+1}$ we have $x=\alpha y-(1-\alpha) \bar{y}$, where $y, \bar{y} \in A_{n+1}^{+}$ and $\alpha \in[0,1]$. Let $A_{n}=\left\{a_{1}^{n}, \ldots, a_{m(n)}^{n}\right\}$. Then there exist $\alpha_{i} \geq 0, \bar{\alpha}_{i} \geq 0$ and $b_{i}, \bar{b}_{i} \in A_{n+1}^{+}\left(a_{i}^{n}\right), i=1, \ldots, m(n)$, with $\sum_{i=1}^{m(n)} \alpha_{i} \leq 1 ; \sum_{i=1}^{m(n)} \bar{\alpha}_{i} \leq 1$ such that

$$
y=\sum_{i=1}^{m(n)} \alpha_{i} b_{i} ; \quad \bar{y}=\sum_{i=1}^{m(n)} \bar{\alpha}_{i} \bar{b}_{i} .
$$

By (7-b) for every $i=1, \ldots, m(n)$ there exists $\lambda_{i}, \bar{\lambda}_{i} \in[0,1]$ such that $\left\|b_{i}-\lambda_{i} a_{i}^{n}\right\|<\varepsilon_{n+1}$ and $\left\|\bar{b}_{i}-\bar{\lambda}_{i} a_{i}^{n}\right\|<\varepsilon_{n+1}$.

Let

$$
z=\sum_{i=1}^{m(n)} \lambda_{i} \alpha_{i} a_{i}^{n} \quad \text { and } \quad \bar{z}=\sum_{i=1}^{m(n)} \bar{\lambda}_{i} \bar{\alpha}_{i} a_{i}^{n}
$$

Then we get

$$
\begin{aligned}
& \|y-z\| \leq \sum_{i=1}^{m(n)}\left\|b_{i}-\lambda_{i} a_{i}^{n}\right\|<m(n) \varepsilon_{n+1} \\
& \|\bar{y}-\bar{z}\| \leq \sum_{i=1}^{m(n)}\left\|\bar{b}_{i}-\bar{\lambda}_{i} a_{i}^{n}\right\|<m(n) \varepsilon_{n+1} .
\end{aligned}
$$

Therefore, setting $b=\alpha z=(1-\alpha) \bar{z} \in \hat{A_{n}}$ from (5) we obtain

$$
\|x-b\| \leq\|y-z\|+\|\bar{y}-\bar{z}\|<2 m(n) \varepsilon_{n+1}<\frac{2 m(n)}{8 m(n)} \delta_{n}=\frac{1}{4} \delta_{n} .
$$

This proves Claim 2-2.

We now define $C$ by the formula

$$
C=\overline{\bigcup_{n=1}^{\infty} \hat{A}_{n}} \subset X .
$$

We shall show that $C$ has the desired property. First we have

2-3. Claim. $\left\|x-\hat{A}_{n}\right\|<\delta_{n}$ for every $x \in C$.

Proof. Let $x \in C$. Take $y \in \hat{A}_{n+k}$ for some $k \in N$ such that $\|x-y\|<\frac{1}{2} \delta_{n}$. Whence using Claim 2-2 we get

$$
\left\|x-\hat{A}_{n}\right\| \leq\|x-y\|+\left\|y-\hat{A}_{n}\right\|<\frac{1}{2} \delta_{n}+\frac{1}{4} \delta_{n}\left(1+\cdots+2^{-k}\right)<\delta_{n} .
$$

The claim is proved.

2-4. Claim. $C$ is compact. 
Proof. Since $A_{n}$ is finite we infer that $\hat{A}_{n}$ is a finite-dimensional compact convex set. Thus by Claim 2-3 $C$ is totally bounded. Therefore, by the completeness of $X, C$ is compact.

We recall that a convex set $A$ of a linear metric space $X$ is admissible if and only if every compact subset of $A$ can be pushed by arbitrarily small maps into finite-dimensional subsets of $A$.

Klee $[\mathrm{K}]$ has shown that every admissible compact convex set is an AR. Using this result we obtain

2-5. Claim. $C$ is an AR.

Proof. Since $C$ is compact it suffices to show that $C$ is admissible. Let $f_{n}=r_{n} \mid C$. Then $f_{n}$ is a retraction from $C$ onto $\hat{A}_{n}$. From (6) and from Claim 2-3 it follows that $\left\|f_{n}(x)-x\right\|<\varepsilon_{n}$ for every $x \in C$. By (5) $\varepsilon_{n} \rightarrow 0$. Since $\hat{A}_{n}$ is finite dimensional we infer that $C$ is admissible. Thus $C$ is an AR by Klee's theorem. The claim is proved.

From (5) and (7) we obtain (see [R1, R2])

2-6. Claim. $C$ contains no extreme points.

Finally we get

2-7. Claim. $C$ is homeomorphic to the Hilbert cube.

Proof. By Claims 2-4-2-6, $C$ is an infinite-dimensional compact convex set which is an AR. Therefore the assertion follows from [DT].

The proof of Theorem 2-1 is finished.

\section{OPEN QUESTIONS}

Since needle point spaces contain compact convex sets with no extreme points it is hoped that they may contain a counterexample to the AR-problem. The following question is of great interest.

3-1. Question. Is every convex set in a needle point space an AR?

We do not know even

3-2. Question. Let $X$ denote the linear metric space constructed by Roberts [R1]:

(i) Is every convex set in $X$ an AR?

(ii) Is every compact convex set in $X$ an AR?

(iii) Is the whole space $X$ an AR?

Let us observe that the AR-problem is still unsolved even among the class of compact convex sets with the fixed point property. Namely, we ask

3-3. Question. Let $X$ be a compact convex set with the fixed point property. Is $X$ an AR?

The whole space $L_{p}, 0 \leq p<1$, are known to be AR's (see [BP, N1, N2]), but it is not known whether convex sets of $L_{p}, 0 \leq p<1$, are AR. Several AR subsets of $M(X)$ (= the space of all measurable functions from $[0,1]$ into a metric space $X$ equipped with the topology of convergence in measure), including $L_{p}, 0 \leq p<1$, have been given in [N1, N2, N3].

\section{Added IN Proof}

After this paper was accepted for publication, J. Mogilski kindly drew our attention to the remark on p. 217 of $A n F$-space sampler by N. J. Kalton, N. 
T. Peck, and J. W. Roberts (London. Math. Soc. Lecture Note Ser., vol. 89, 1984), of which we were unaware, which claims (without proof) that all the examples constructed by Roberts' method of needle point spaces have the fixed point property. The AR-property, however, is much harder than the fixed point property even for compact convex sets (see Question 3-3).

\section{REFERENCES}

[BP] C. Bessaga and A. Pelczynski, Selected topics in infinite dimensional topology, PWN, Warszawa, 1975.

[DG] J. Dugundji and A. Granas, Fixed point theory. I, PWN, Warszawa, 1982.

[DT] T. Dobrowolski and H. Torunczyk, Separable complete ANR's admitting a group structure are Hilbert manifolds, Topology Appl. 12 (1981), 229-235.

[G] R. Geoghegan, Open problems in infinite dimensional topology, Topology Proc. 4 (1979), 287-333.

[K] V. L. Klee, Shrinkable neighbourhoods in Hausdorff linear spaces, Math. Ann. 141 (1960), 281-285.

[KM] M. G. Krein and D. P. Milman, On extreme points of regular convex sets, Studia Math. 9 (1940), 133-138.

[KP] N. J. Kalton and N. T. Peck, A re-examination of Roberts' example of compact convex sets with no extreme points, Math. Ann. 253 (1980), 89-101.

[N1] Nguyen To Nhu, Investigating the ANR-property of metric spaces, Fund. Math. 124 (1984), 243-254.

[N2] _ Remarks on measurable function spaces, Acta Math. Vietnam 12 (1987), 85-92.

[N3] - The group of measure preserving transformations of the unit interval is an absolute retract, Proc. Amer. Math. Soc. 110 (1990), 515-522.

[R1] J. W. Roberts, A compact convex set with no extreme points, Studia Math. 60 (1977), 255-266.

[R2] Pathological compact convex sets in the spaces $L_{p}, 0 \leq p<1$, The Altgeld Book, Univ. of Illinois, 1976.

[Re] S. Rolewicz, Metric linear spaces, PWN, Warszawa, 1972.

[Ru] W. Rudin, Functional analysis, McGraw-Hill, New York, 1973.

Institute of Mathematics, P. O. Box 631, Bo Ho, Hanol, Vietnam 